\title{
A randomized controlled trial evaluating a pain assessment and management program for respite workers supporting children with disabilities part one: Pain-related knowledge and perceptions
}

Lara M. Genik, C. Meghan McMurtry, Elisabeth L. Aerts, Paula C. Barata, Chantel C. Barney, Stephen P. Lewis, Ian Newby-Clark

Genik, L. McMurtry, M. Aerts, E. Barata, C. Stephen, B. Newby-Clark, I. 2020. A randomized controlled trial evaluating a pain assessment and management program for respite workers supporting children with disabilities part one: Pain-related knowledge and perceptions. American Journal on Intellectual and Developmental Disabilities. [Manuscript accepted].

\section{Author Note}

Lara M. Genik, MA, PhD Candidate, lgenik@uoguelph.ca, Department of Psychology, University of Guelph; C. Meghan McMurtry, PhD, C. Psych, cmcmurtr@uoguelph.ca, Department of Psychology, University of Guelph, Clinical Psychologist, Pediatric Chronic Pain Program, McMaster Children's Hospital, Associate Scientist, Children’s Health Research Institute, Adjunct Researcher, Department of Pediatrics, Western University; Elisabeth L. Aerts, BSc, aertse@mail.uoguelph.ca, Department of Psychology, University of Guelph; Paula C. Barata, PhD, pbarata@uoguelph.ca, Department of Psychology, University of Guelph; Chantel C. Barney, PhD, cbarney@gillettechildrens.com, Gillette Children’s Specialty Health Care, Department of Educational Psychology, University of Minnesota; Stephen P. Lewis, PhD, slewis03@uoguelph.ca, Department of Psychology, University of Guelph; Ian Newby-Clark, PhD, inewby@uoguelph.ca, Department of Psychology, University of Guelph. Manuscript submitted as an original article. 
This research was funded by a Canadian Institutes of Health Research Doctoral Research Award, the Society of Pediatric Psychology's Marion and Donald Routh Student Research Grant, and the Annual Dr. Benjamin Goldberg Research award, and was conducted as part of a doctoral dissertation research project within the Pediatric Pain, Health and Communication (PPHC) Lab under the supervision of McMurtry. Genik is a trainee within the Pain in Child Health Strategic Training Program. Components of the work in this manuscript have been previously presented at the International Symposium on Pediatric Pain (June, 2019). The authors have no conflicts of interest to declare. Special thanks to Andrea Cross and Dr. Peter Rosenbaum for their willingness to assist with control group facilitation for the RCT.

Correspondence concerning this article should be addressed to Lara Genik, Department of Psychology, University of Guelph, Guelph, ON N1G 2W1. Telephone: 519-824-4120 ext. 52342 Email: lgenik@uoguelph.ca 


\begin{abstract}
Pain is common for children with intellectual and developmental disabilities (I/DD), yet specialized caregiver-based education is lacking. Objectives: This parallel group RCT tested effectiveness of the Let's Talk About Pain training on respite workers' (RW) pain-related knowledge and feasibility-confidence-skill ratings using between-group (pain vs. control) and within-group (pain training only) analyses. Methods: Fourteen children's respite organizations were randomized using sequentially numbered, opaque, sealed envelopes to receive pain or control training. Researchers were blind until randomization; allocations were not explicitly revealed to organizations and participants. Participants $\left(n_{\text {intervention }}=66 ; n_{\text {control }}=92\right)$ underwent a 3-3.5 hour training and completed pain-related knowledge measures and feasibility-confidenceskill ratings at pre, post and 4-6 week follow-up. Intention-to-treat $\left(n_{\text {intervention }}=65 ; n_{\text {control }}=92\right)$ and per-protocol $\left(n_{\text {intervention }}=26-38, n_{\text {control }}=40-57\right)$ analyses were conducted. Results: Participants receiving pain training demonstrated (a) significantly higher pain knowledge and feasibility-confidence-skill ratings at post and follow-up versus control group and (b) significant increases in knowledge from pre-post. Despite a small decrease in mean scores, significant gains in knowledge and confidence-skill ratings were maintained from post-follow-up. Discussion: Results demonstrated improvements in RW knowledge and feasibility-confidence-skill ratings. This represents a promising step towards enhancing pain-related care provided to children with I/DD.
\end{abstract}

Keywords: pain; children; intellectual and developmental disabilities; education; respite workers 


\section{Introduction}

Pain is a common experience for children with intellectual and developmental disabilities (I/DD) that negatively affects their adaptive functioning (e.g., daily living skills, communication, social skills; Breau, Camfield, McGrath, \& Finley, 2003; Breau, Camfield, McGrath, \& Finley, 2007). Numerous factors, including communication difficulties, cognitive deficits, and unique pain behaviors contribute to challenges with pain assessment and management (Carter, McArthur, \& Cunliffe, 2002; Doody \& Bailey, 2017). The majority of research to date has focused on developing assessment tools for primary caregivers and health care providers (e.g., Non-Communicating Children's Pain Checklist; Breau, Camfield, McGrath, \& Finley, 2002). There has been limited development of targeted educational programs for key staff in residential and school settings (Mackey \& Dodd, 2010; Quinn \& Smolinski, 2017). Participants receiving education in these studies have reported: (a) altered attitudes such as better awareness that people with I/DD do not have higher pain thresholds (Mackey \& Dodd, 2010); (b) less difficulty assessing pain (Mackey \& Dodd, 2010; Quinn \& Smolinski, 2017); and (c) intent to change practice standards (Quinn \& Smolinski, 2017).

Respite care refers to temporary care of a child with a disability which aims to provide a parent or primary caregiver a break from the demands of caring for their child and their needs (McConkey, Kelly, \& Craig, 2011). Notably, respite care is identified by families of children with I/DD as a critical, high-in-demand support service (McConkey et al., 2011; Douma, Dekker, \& Koot, 2006; Robertson et al., 2011). Yet, many respite workers have not received specialized pain education, and may hold inaccurate pain-related beliefs 
Pain program to address this gap. Immediately following the training, participating respite workers $(N=50)$ demonstrated significant increases in pain-related knowledge (large effect sizes $r=0.81-0.88$ ); respite workers also provided higher ratings of the feasibility of pain assessment and management, as well as their confidence and skill in these domains (herein referred to as feasibility-confidence-skill ratings; moderate to large effect sizes $r=0.41-0.70$; 2018). Although promising, this pilot did not include a comparison group or follow-up time point; hence, the present study was designed to address these limitations.

As part of a larger randomized controlled trial (RCT) using a parallel two group design (ClinicalTrials.gov identifier: NCT03421795) with pre, post and four to six week follow-up measures, the objectives of this study were to systematically test the effectiveness of the Let's Talk About Pain program on respite workers' (a) pain-related knowledge (primary outcome), and (b) feasibility-confidence-skill ratings (secondary outcome) for children with I/DD. We hypothesized that at post and follow-up, respite workers who completed a pain training would demonstrate significantly higher pain-related knowledge scores and feasibility-confidence-skill ratings compared to those receiving the control training. We also expected that participants assigned to the pain training group would demonstrate significant increases from pre to post and maintenance of these gains from post to follow up for all outcomes.

\section{Materials and Methods}

Data were collected as part of a multi-center parallel two group (wait-list control) RCT approved by our research ethics board. The RCT consisted of two components. In this manuscript, the quantitative analyses of the impact of the Let's Talk About Pain program on participants' pain knowledge and perceptions of feasibility, confidence and skill are described. 
Part two appears in a separate manuscript

submitted) and focuses on the qualitative analysis of participants' Let's Talk About Pain training evaluations, and the impact of this training on their use of pain-related strategies. Separate manuscripts for these outcomes allow for adequate discussion and exploration of the results and their implications. The same group of participants are represented in both manuscripts; however, the data in these two publications are distinct except for participant demographics.

Only the methods relevant to the present article are described below. A complete version of the RCT protocol is available 2020). The authors assume full responsibility for consistency between this report and the study protocol, as well as the correctness and completeness of the trial data.

\section{Participant Recruitment and Randomization}

Organization Recruitment. Organization-based recruitment occurred in collaboration with children's respite organizations in Ontario. Ninety-five organizations were assessed for eligibility (i.e., whether they offered respite services for children with I/DD; see 2020) and contacted by telephone and/or email. Upon contact, organizations were informed about the research study, which provided the organizations an opportunity to receive one to two free training sessions for respite staff.

After agreeing to host a training session, organizations were randomly assigned to either the pain training intervention condition or the control condition with an opportunity to schedule the pain training after follow-up. Assignments were completed using a permuted block design (total block size: 14; allocation ratio: 1:1). Sequentially numbered, opaque, sealed envelopes were created by a research assistant who was not involved in the randomization process. Organizations were not told to which condition they were allocated, and primary researchers 
remained blind until after randomization occurred (for details see

randomization, organizations were given information about the training and study component to circulate in advance to staff.

Individual Participant Recruitment. At the beginning of the initial training date for each organization, the optional study component was discussed in detail with staff. The study purpose was described as examining the impact of training on respite workers' knowledge about caring for children with I/DD. Eligible staff were proficient in English, at least 18 years of age, and employed at the participating respite organization providing respite to children with I/DD. They could not hold upper management positions or participate more than once in the study if employed in more than one participating organization.

\section{Training Interventions}

General Training Characteristics and Training Fidelity. General training characteristics of both groups were informed by the results of $\square$ and colleagues' pilot study of the Let's Talk About Pain program ( 2018). Both the intervention and control training were single training sessions that lasted three to three and a half hours in length. They used a standardized Power-Point presentation with notes and included several interactive components (e.g., group discussions, case studies). Participants also received printouts of the slides and relevant resources. The primary investigator conducted all intervention trainings and a different facilitator from an external organization completed the control trainings; both had prior experience facilitating their corresponding trainings and applied experience in the field. Trainings were provided in-person within participating organizations' spaces. Participants were given the opportunity to ask questions throughout the training, which allowed for some tailoring to the organizations' interests and needs. 
During each training session, a fidelity checklist was completed by a research assistant. The purpose of the checklist was to formally document similarities regarding training length, break length, and group size across conditions, and ensure that key points of information were covered in the intervention training and not covered in the control training. The pain training facilitator also had access to this checklist during each training to help ensure the pertinent information was covered. See Table 1 for a summary of fidelity data. Of note, there was a significant difference (see Table 1) between the length of the intervention and control training, such that the intervention training was on average 18 minutes longer.

Let's Talk About Pain Training. As in the pilot study 2018), the training aimed to increase knowledge and improve perceptions about pain in children with I/DD. Developed using stakeholder input, information from chapter 43 of the International Association for the Study of Pain's Core Curriculum (Charlton, 2005), and research literature, the training provided education relevant to respite contexts about: what pain is, pain expression, pain assessment, and pain management (2018). Training materials and a full training outline are available from the corresponding author upon request.

Control Training. The training provided to control groups focused on a family-centred care approach intended to improve the quality of life of children with I/DD. Specifically, this training communicated the six 'F-words' of childhood disability (function, family, fitness, fun, friends, future; Rosenbaum \& Gorter, 2012) and discussed ways to implement these principles into respite programming. This training reflects the World Health Organization's (2001) International Classification of Functioning, Disability and Health Framework. Thus, like the pain training, the control training was relevant but novel for respite settings.

\section{Procedures}


Initial Data Collection. After providing written informed consent, participants from both the intervention and control groups were asked to complete a series of pre-intervention paperbased questionnaires in person including: (a) a demographics questionnaire; (b) a pain-related knowledge measure specific to individuals with I/DD; and (c) feasibility-confidence-skill ratings for pain assessment and management. Immediately following consent and pre-intervention questionnaires, participants completed either the intervention or control training session. Immediately following each session, participants were asked to answer post-intervention paperbased questionnaires in person, which were identical to (b) and (c) previously described. All participants received a folder with a copy of the slides, relevant resources, a notepad/pen set, refreshments during the break, and an opportunity to enter a \$20 gift card draw (odds of winning: 24 to 1$)$.

Follow-Up Data Collection. Approximately four to six weeks following initial data collection, the researchers collected pain knowledge and feasibility-confidence-skill ratings using paper-based questionnaires from both groups in person. All follow-up participants received a certificate of completion, another opportunity to enter a \$20 gift card draw (odds of winning: 24 to 1), and \$20 cash. After follow-up data collection, organizations in the control condition were given the opportunity to complete the Let's Talk About Pain training program at a time convenient for the organization.

\section{Measures}

Organization and Participant Demographics Questionnaires (Pre). In order to gather information about the host organizations, an organization staff member in an upper leadership position was asked to complete a brief questionnaire about the organization itself. This included 
questions regarding the type of respite programming that staff are involved in, the types of children staff work with, and staff to child ratios. Individual participants self-reported general demographic information (e.g., age, gender), information about their experience with children with I/DD, and information about any previous pain-related education completed.

Questionnaire for Understanding Pain in Individuals with Intellectual Disabilities Caregiver Version Revised (QUPID-CR; Pre, Post, Follow-Up). The QUPID-CR was used to assess participants' knowledge regarding: general pain, pain assessment, and pain management in individuals with DD. The QUPID-CR is a 39-item, empirically-informed true/false and multiple choice questionnaire; one "point" is given for each correct response to compute a total knowledge score out of 39. The QUPID-CR is available from authors upon request. The QUPIDCR is a revised version of the initial QUPID-C, which was responsive to knowledge change in an RCT with undergraduate students; 2017) and in and colleagues' Let's Talk About Pain pilot study 2018). Revisions to the original QUPID-C consisted of alterations to seven of the original items which performed poorly according to completed item analyses , in preparation). Four new items were also added to the QUPID-CR assessing knowledge regarding pain science and theory (e.g., gate control theory of pain in preparation). Initial item analyses and responsivity testing for the QUPID-CR support the use of the QUPID-CR ( in preparation).

Feasibility-Confidence-Skill Ratings (Pre, Post, Follow-Up). Participants provided selfreport ratings on 6 items: the feasibility of pain assessment and pain management $(0=N o t$ Feasible At All, 10 = Highly/Extremely Feasible); their confidence in (pain assessment, pain management $(0=$ Not Confident At All, $10=$ Highly/Extremely Confident $)$; and their skill in pain 
assessment and management; $(0=$ Not Skilled At All, $10=$ Highly/Extremely Skilled $)$ for children with I/DD. Therefore, there were 6 ratings collected at each time point. These ratings showed responsivity to training in a pilot $\mathrm{RCT}$ with undergraduate students 2017), and in and colleagues' pilot study for the Let's Talk About Pain training program , 2018).

\section{Data Collection Summary}

Figure 1 represents a flow diagram of host organization and participant enrollment/allocation, participation, and analysis phases. Individual participant recruitment and data collection occurred across 14 organizations with children's respite services in southwestern, central, northern and eastern Ontario from May 2017 to August 2018. A total of 158 respite workers' data were included in the analyses. All participants in the control group $(n=92 ; 100 \%)$ and 65/66 participants in the intervention group (95.45\%) completed the training intervention in its entirety. The response rate at follow-up included $60.60 \%(n=40)$ participants in the intervention group and $67.39 \%(n=62)$ participants in the control group. There were no crossovers between groups during the study period as each organization was only randomly assigned to one condition, and all participants were employed in only one participating organization. Five of the seven organizations who were allocated to the control group held the pain training after study completion.

\section{Statistical Analysis}

\section{Analysis of Demographic Data}

Frequency and descriptive analyses were used to analyze participants' demographic data including age, gender, ethnicity, number of years employed in respite settings, experience with children with I/DD, and exposure to pain-related training. When participants left demographic questions blank (e.g., gender, age, frequency of interaction with children with I/DD), they were 
excluded from those specific analyses only. Demographic data were compared using independent samples t-tests and chi-square analyses to determine whether the groups significantly differed on age, gender, years working with children with I/DD, or degree of involvement with children with I/DD.

\section{Analysis of Primary and Secondary Outcomes}

A series of seven 2 (group: treatment, control) x 3 (time: pre, post, follow-up) mixed analyses of variance (ANOVA) were conducted. The dependent variables for the mixed ANOVAs were the participants' pain-related knowledge scores (primary outcome measure), and feasibility-confidence-skill ratings (secondary outcome measures). Importantly, there are two common approaches used when conducting analyses for RCT data: 1) intention-to-treat (ITT), which includes all participants who provided data and uses approaches such as imputation to manage missing data (Armijo-Olivo, Warren, \& Magee, 2009), and 2) per-protocol (PP) which includes only participants who completed all aspects of the study. Advantages and disadvantages exist with each approach; however, ITT can provide a more conservative estimate of treatment effect, and PP can better reflect treatment differences when participants complete the study protocol (Gupta, 2011). In order to benefit from each of these advantages, ITT was considered the primary analysis approach while PP analyses were considered complementary (Armijo-Olivo et al., 2009; Gupta, 2011).

ITT. When using ITT, there has been some concern that it may become more about the treatment prescribed as opposed to the treatment received if participants withdraw prior to receiving the intervention (Armijo-Olivo et al., 2009). As such, the one participant who did not complete the full training intervention was excluded from ITT analyses. Missing data analyses were then conducted to determine the nature of all other missing data from the QUPID-CR 
questionnaire as well as the feasibility-confidence-skill ratings, and the appropriate imputation method to be applied.

Participants were categorized as missing data on the QUPID-CR if they completed the corresponding time point and had as little as one missing response on the questionnaire. Specifically, the percentage of participants who selected multiple responses, gave unclear responses, or were otherwise missing one or more QUPID-CR question responses at the pre, post, and follow up time periods were $22.60 \%, 10.96 \%$, and $10.78 \%$, respectively. Of importance, only three participants in the pre period and two in the post period had 4-7 questions considered missing on the QUPID-CR. All other participants with some form of missing data were missing one to three questions. The percentage of participants missing data on at least one of the six feasibility-confidence-skill ratings ranged from $0.64 \%$ - and $12.74 \%$ across time periods.

Results using Little's MCAR test to further explore missing QUPID-CR and feasibilityconfidence-skill data suggested that data on these measures across time points were considered missing completely at random ( $p$ 's range from $.121-.885)$. The only exception was for the QUPID-CR post data time point, $x^{2}(540)=613.52, p<.001$. Additional comparison analyses for QUPID-CR post data were therefore conducted between participants with and without missing data, which were non-significant ( $p$ 's range from .131 to .939). We can therefore conclude that this QUPID-CR post data is missing at random. To account for the aforementioned patterns, a conservative approach was applied to all QUPID-CR and feasibility-confidence-skill rating data, labelling it as missing at random. This implies that the data were, at a minimum, 'randomly distributed within one or more subsamples' (Armijo-Olivo et al., 2009). 
Due to the high attrition rate of participants at follow-up, a series of comparison analyses were also conducted specific to participants who did and did not complete follow up measures. Demographic characteristics (i.e., age, years working with children with I/DD, degree of involvement with children with I/DD, gender), participant condition (i.e., intervention vs. control) and baseline scores (i.e., QUPID-CR, feasibility-confidence-skill ratings) were compared using independent samples t-tests and chi square tests. There were no significant differences ( $p$ 's range from .129 - .963).

When imputing data, SPSS creates several data sets that estimate how the data set may have looked if there were no missing values. In this case, the five final iterations of these data sets are presented. As SPSS does not create and use a pooled estimate for mixed ANOVAs, these analyses were conducted on each of the five final iterations of imputed data, with ranges of values reported. Analyses in which pooled estimates are available (i.e., follow-up one-way ANOVAs and paired samples t-tests) are reported with specific estimates rather than ranges.

$\boldsymbol{P P}$. Figure 1 indicates the total number of participants per group included in each of the per protocol analyses. A modified PP approach was used for these complementary analyses, such that for participants with missing data on a given item needed for a specific analysis, their data were excluded from the corresponding PP analysis.

\section{Results}

\section{Host Organizations and Participants}

Table 2 provides demographic information about the participating host organizations which spanned geographically across Ontario, Canada. A total of 158 respite workers $\left(M_{\text {age }}=\right.$ 30.67; $S D=8.62)$ consisting of $87.97 \%$ women $(n=139)$ and $12.03 \%$ men $(n=19)$ participated (83.40\% European/White; 5.70\% Black/African/Caribbean; 4.50\% South and Southeast Asian). Consistent with a-priori power analyses using G*Power 3 (Faul, Erdfelder, Lang \& Buchner, 
2007) and effect sizes from and colleagues' pilot study 2018), this sample size is sufficient to investigate the effectiveness of the Let's Talk About Pain training using mixed ANOVA at power of 0.95 and an alpha of 0.05 . All participants provided respite to children with I/DD in at least one of the following settings: in the family home $(n=72 ; 46.15 \%)$, in respite workers' own homes $(n=33 ; 21.15 \%)$, in the community $(n=119 ; 76.28 \%)$, and at a group home, respite home, residential facility or specialized respite programming $(n=130$, $83.33 \%)$. Seven $(4.48 \%)$ participants also endorsed providing respite in other settings not listed above such as in hospital or clinical settings. The demographic composition of the intervention and control groups are summarized in Table 3. There were no significant differences between (a) demographic characteristics of the intervention and control groups (see Table 3), and (b) baseline scores and ratings on outcome measures (see Table 4).

\section{ITT Analyses}

Pain Knowledge (Primary Outcome). Table 4 shows the range of means and standard deviations for ITT analyses for all outcome measures at all time points. ITT analyses using a mixed ANOVA denoted a significant interaction effect of training type (pain training, control training) with time (pre, post, follow-up) for participants' pain-related knowledge (see Table 5, a large effect). Follow-up analyses using pooled ITT data show support for between-group hypotheses, such that those receiving the pain training had significantly higher pain knowledge scores at post than those receiving the control training Welch's $F(1,845.05)=392.84, p<.001$,

$\omega^{2}=0.26$ (a large effect; Kirk, 1996) and at follow-up, Welch's $F(1,873.69)=225.17, p<.001$, $\omega^{2}=0.18$ (a large effect; Kirk, 1996). Within-groups hypotheses were also partially supported. Those who received the pain training showed significant increases in pain-related knowledge from pre to post, $t(55)=8.71, p<.001, d=1.08$ (a very large effect; Sawilowsky, 2009). 
Although there was a significant decrease in knowledge from post to follow-up, $t(112)=3.62, p$ $<.001, d=.45$ (a medium effect; Cohen, 1988), participant knowledge scores remained significantly higher at follow-up compared to pre, $t(67)=6.36, p<.001, d=.79$ (a large effect; Cohen, 1988). These results are displayed in Figure 2.

Feasibility, Confidence and Skill Ratings (Secondary Outcomes). Significant interaction effects of training type by time periods were demonstrated for the following ratings: Assessment Confidence, Assessment Skill, Management Confidence, Management Skill (see Tables 4 and 5). Follow up analyses using ITT data demonstrated that all six ratings were significantly higher for participants who completed the pain training compared to the control group at both post and follow up time points (see Table 6). For the within pain group analyses and consistent with pain-related knowledge findings, all six feasibility-confidence-skill ratings increased significantly immediately following the training (post). At follow-up, ratings of confidence and skill for both assessment and management increased significantly from pre to follow-up, but assessment and management feasibility ratings were not significantly different from pre to follow-up (see Table 7 and Figure 3)

\section{PP Analyses}

Analyses completed for PP data followed the same results pattern as described above, except for participants' feasibility ratings. Specifically, there were no significant group by time interaction effects for assessment nor management feasibility ratings when using PP analyses.

\section{Discussion}

For children with I/DD, pain is a common experience that is frequently under-recognized and under-managed (Breau et al., 2003; McGuire, Daly \& Smyth, 2010). The mismanagement of pain in children with I/DD has important implications for their quality of life, sleep, and 
functioning (Breau et al., 2007; Breau \& Camfield, 2011; Tudor, Walsh, Mulder, \& Lerner, 2015). It is critical that secondary caregivers, including respite workers, can properly assess and manage pain in children with I/DD. Importantly, respite workers may hold inaccurate beliefs that can influence the care they provide; these workers are also lacking opportunities to receive specialized pain-related training 2017). Let's Talk About Pain was developed and successfully piloted with intent to fill this need; however, the pilot study was limited in its lack of comparison group and cross-sectional design ( 2018). The current RCT addressed these limitations by providing a more rigorous and longitudinal examination of the effects of Let's Talk About Pain via data collection over three time points, and utilization of a control group.

\section{Pain Knowledge}

As hypothesized, pain-related knowledge of the intervention group increased immediately following the training (very large effect) and was higher than the control group (large effect). The improvement in pain knowledge was observed despite already strong baseline knowledge in both groups. This large increase in knowledge is consistent with $\square$ and colleagues' (2018) Let's Talk About Pain pilot findings as well as similar outcomes in other pain-related intervention studies (_2017; Zhang et al., 2008). Increases in pain knowledge demonstrated by the intervention group remained higher than the control group at 4-to-6 week follow-up (large effect) and their own baseline scores, but decreased from an average of approximately 33 points to 31 points out of 39 on the knowledge measure (medium effect). Although this training may be effective in improving knowledge of respite workers, at least some degree of knowledge decay may occur overtime. The decrease in knowledge may suggest factors such as implementation and on-the-job experiences influence knowledge 
retention. For example, failure to sufficiently use these new skills and knowledge following training may impact one's ability to consolidate it; data exploring the training's impact on strategy use are described in a separate manuscript submitted).

Although more training sessions may have potential benefit in maintaining increases in pain-related knowledge, the single-session training format in the current study was chosen based on feedback from stakeholders regarding feasibility (ـ2018). Organizations may have limited resources for continued training or boosters facilitated by outside personnel. The Trainthe-Trainer model is an example of an efficient and effective knowledge dissemination approach that could help to mitigate some of these challenges (e.g., Anderson \& Taira, 2018; Marks, Sisirak \& Chang, 2013). For example, creating 'pain champions' who could take knowledge and skills back to their organizations to implement these with staff may improve knowledge implementation.

\section{Feasibility-Confidence-Skill Ratings}

The pattern of results for participants' self-reported feasibility-confidence-skill ratings at pre, post and follow-up suggest educational programming can alter staff perceptions. Like pain knowledge above, the increases in confidence and skill ratings for assessment and management remained significantly higher than pre-scores. Consistent with previous research 2018), the patterns of responses between confidence and skill were very similar, suggesting that these constructs may overlap in some way. Unlike confidence and skill ratings, the ratings for feasibility of assessment and management were not significantly higher than pre-scores at follow-up. This makes sense, as one could perceive something as feasible but be lacking in confidence or skill, and vice versa. Further, feasibility may be perceived as outside of one's control (e.g., child, family, or organization factors may make aspects of assessment or 
management less feasible), whereas skill and confidence may be perceived as within a caregiver's control (e.g., can get more education, practice using skills). Consideration of each of these ratings separately rather than collapsed is therefore important in understanding these selfreport constructs.

First, although intervention participants' ratings of pain assessment and management feasibility improved from pre to post, the effect size was small. These ratings again decreased at follow-up such that there was no difference between pre and follow-up scores. The limited change in feasibility may reflect implementation-related challenges often experienced in organizational contexts, particularly when only front-line staff are included in the trainings. For example, while school nurses struggled to implement new knowledge despite intent in one study related to pain in children with I/DD (Quinn \& Smolinski, 2017), 95\% of participants in another study believed they could implement new practices in residential care settings for people with I/DD when the training was designed for managers (Mackey \& Dodd, 2010). Further, several inherent challenges for people with I/DD may continue to limit the feasibility of pain assessment and management even when caregivers have adequate knowledge and skills.

Second, despite the increase in self-reported confidence following the intervention, confidence ratings of both groups at all time points ranged from 5.95-7.68 (moderate to moderately-high). These ratings may reflect an awareness of the complexities and challenges associated with pain assessment and management in this population; ratings of 'extreme' confidence may not actually be ideal. Concerns in other health-care contexts have been raised about being 'overconfident' versus 'appropriately confident' and its implications for clinical practice, such as a failure to learn from experiences or to consider alternatives (Naguib et al., 
2019). Thus, it may be important to consider what 'appropriate' confidence might look like in respite settings and how to achieve this.

Finally, participants rated their perceived skill in pain assessment and management. Assuming confidence and skill are related, one may also wish to consider the implications of over- versus appropriate confidence in relation to skill. Indeed, one's perceptions may not always map onto their actual skill in a domain (Glakchen \& Bookbinder, 2001) and this could impact care. For example, it is possible that respite workers may believe they have skill in a strategy but may inappropriately apply it. In operationally defining 'skill', one could more objectively compare perceived and actual skill in care settings. This may be a more appropriate way to determine skill and develop an understanding of the ways that one's skill perceptions may be similar or different from observed skill.

\section{Strengths and Limitations}

To our knowledge, this was the first RCT to systematically evaluate a specialized pain training program for respite workers of children with I/DD. Designed using extant research literature and the International Association for the Study of Pain's Core Curriculum (Charlton, 2005), data were collected over multiple time points and compared with a group receiving related but distinct training on family-centered care. Recruitment proceeded through multiple children's respite organizations supporting children with varying needs. The use of CONSORT guidelines, clear reporting and trial registration should allow for replication and implementation of this training. Further, the structure and content of this training may lend itself well to the development of a more manualized training or a train-the-trainer approach that could be shared with key staff within children's respite organizations. 
Although organizations and participants were not explicitly told of the study condition they were assigned to or hypotheses, it is possible that they were able to infer this information based on the provided information. Further, self-report measures were used as a key means of data collection which can lead to bias, and potential overestimation or reporting. For example, those in the pain training condition may have rated their perceived confidence in pain assessment as higher regardless of how they felt after the training (although notably this did not occur for feasibility). A large percentage of participants were lost to follow-up. Despite the presence of missing data across time points, missing data was approached in a very conservative way and missing data on relevant dependent variables was generally below $20 \%$ as recommended in the literature (Armijo et al., 2009); the exception was the pre time point for the QUPID-CR where 22.6\% was missing. These are not uncommon challenges in RCTs and relevant missing data analyses indicated that all missing data seemed to be 'missing at random'. Further, analyses using ITT and PP methodology were conducted and the results were identical.

\section{Future Directions and Conclusions}

This study allowed for rigorous examination of Let's Talk About Pain's impact on respite worker's pain knowledge and feasibility-confidence-skill ratings. Consistent with $\_$and colleagues' pilot study (ـ2018), respite workers' knowledge and feasibilityconfidence-skill ratings significantly increased immediately following completion of a pain training. Pain knowledge and confidence-skill ratings from the intervention group were also significantly higher than the control group at post and follow-up. Respite workers who completed the pain training experienced some knowledge decay at a follow-up time point as early as four to six weeks following training completion. Future work should explore the type of knowledge loss that occurs over time, and its impact on care. Finally, while the program in its 
current state has been designed for respite workers, this type of education could also benefit other similar secondary caregivers (e.g., educational assistants), or used as a model for the development of additional training programs to benefit other professions who support children with I/DD (e.g., physical therapists). Research in pain and I/DD populations is scarce, particularly with respect to studies related to knowledge translation and application. These efforts are critical to share knowledge with those who need it to better meet the needs of children with I/DD. 


\section{References}

Anderson, C. R., \& Taira, B. R. (2018). The train the trainer model for the propagation of resuscitation knowledge in limited resource settings: A systematic review. Resuscitation, $127,1-7$.

Armijo-Olivo, S., Warren, S., \& Magee, D. (2009). Intention to treat analysis, compliance, dropouts and how to deal with missing data in clinical research: A review. Physical Therapy Reviews, 14(1), 36-49.

Breau, L. M., \& Camfield, C. S. (2011). Pain disrupts sleep in children and youth with intellectual and developmental disabilities. Research in Developmental Disabilities, $32(6), 2829-2840$.

Breau, L. M., Camfield, C. S., McGrath, P. J., \& Finley, G. A. (2003). The incidence of pain in children with severe cognitive impairments. Archives of Pediatrics \& Adolescent Medicine, 157(12), 1219-1226.

Breau, L. M., Camfield, C. S., McGrath, P. J., \& Finley, G. A. (2007). Pain's impact on adaptive functioning. Journal of Intellectual Disability Research, 51(2), 125-134.

Breau, L. M., McGrath, P. J., Camfield, C. S., \& Finley, G. A. (2002). Psychometric properties of the non-communicating children's pain checklist-revised. Pain, 99(1-2), 349-357.

Carter, B., McArthur, E., \& Cunliffe, M. (2002). Dealing with uncertainty: Parental assessment of pain in their children with profound special needs. Journal of Advanced Nursing, 38(5), 449-457.

Charlton, J.E. (2005). Core curriculum for professional education in pain. IASP Press. 
Cohen, J. The analysis of variance and covariance: Statistical power analysis for the behavioral sciences. Statistical power analysis for the behavioural sciences, second edition. United States of America. Lawrence Arlbaum Associates; 1988. pp. 1-559.

Doody, O., \& E. Bailey, M. (2017). Pain and pain assessment in people with intellectual disability: Issues and challenges in practice. British Journal of Learning Disabilities, $45(3), 157-165$.

Douma, J. C. H., Dekker, M. C., \& Koot, H. M. (2006). Supporting parents of youths with intellectual disabilities and psychopathology. Journal of Intellectual Disability Research, $50(8), 570-581$.

Faul, F., Erdfelder, E., Lang, A. G., \& Buchner, A. (2007). G* Power 3: A flexible statistical power analysis program for the social, behavioral, and biomedical sciences. Behavior Research Methods, 39(2), 175-191. 

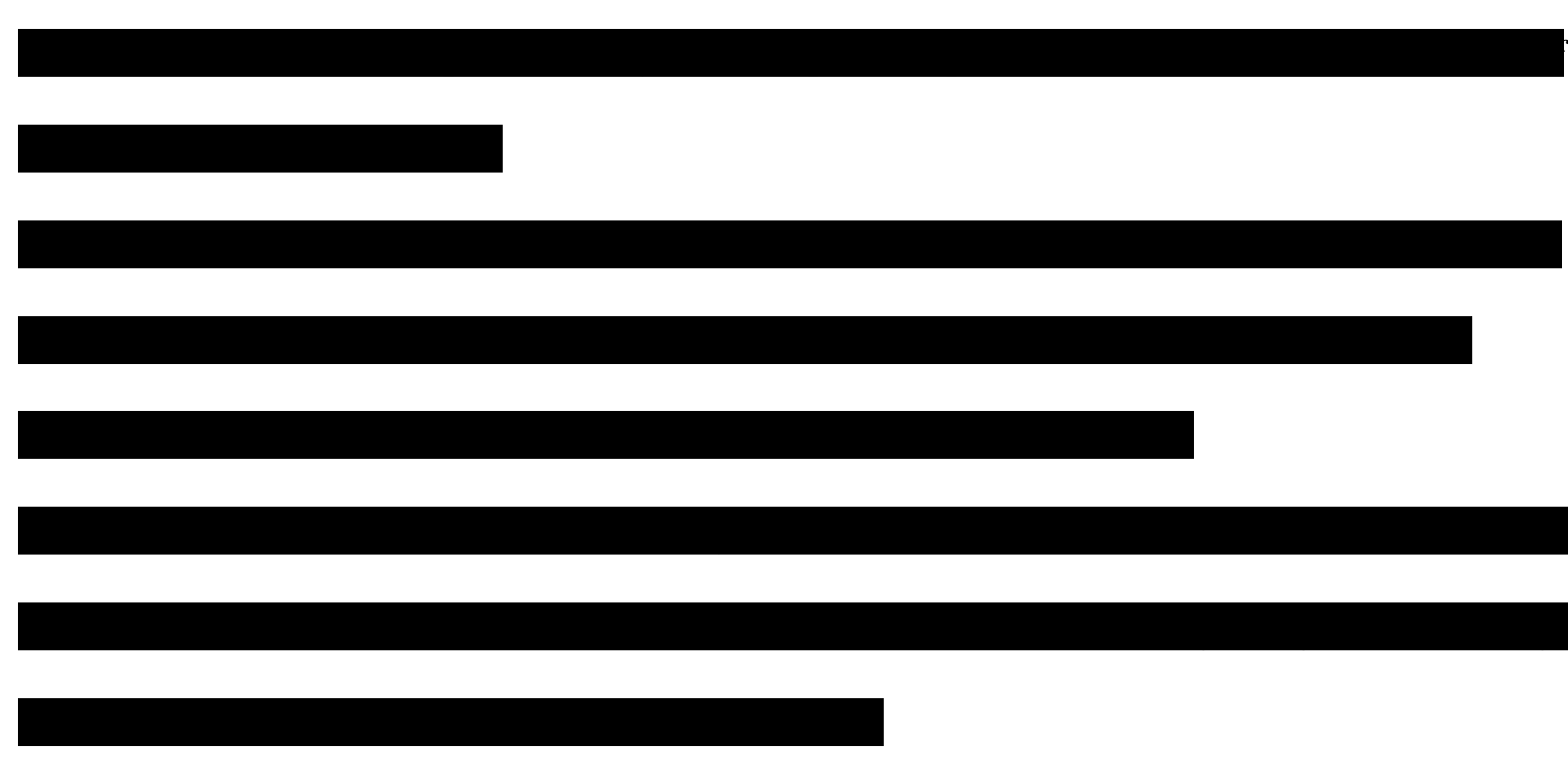

Glajchen, M., \& Bookbinder, M. (2001). Knowledge and perceived competence of home care nurses in pain management: A national survey. Journal of Pain and Symptom Management, 21(4), 307-316.

Gupta, S. K. (2011). Intention-to-treat concept: A review. Perspectives in Clinical Research, 2(3), 109.

Kirk, R. E. (1996). Practical significance: A concept whose time has come. Educational and Psychological Measurement, 56(5), 746-759.

Mackey, E., \& Dodd, K. (2011). Evaluation and effectiveness of pain recognition and management training for staff working in learning disability services. British Journal of Learning Disabilities, 39(3), 243-251.

Marks, B., Sisirak, J., \& Chang, Y. C. (2013). Efficacy of the HealthMatters program train-thetrainer model. Journal of Applied Research in Intellectual Disabilities, 26(4), 319-334. 
McConkey, R., Kelly, F., \& Craig, S. (2011). Access to respite breaks for families who have a relative with intellectual disabilities: a national survey. Journal of Advanced Nursing, 67(6), 1349-1357.

McGuire, B. E., Daly, P., \& Smyth, F. (2010). Chronic pain in people with an intellectual disability: Under-recognised and under-treated?. Journal of Intellectual Disability Research, 54(3), 240-245.

Naguib, M., Brull, S. J., Hunter, J. M., Kopman, A. F., Fülesdi, B., Johnson, K. B., \& Arkes, H. R. (2019). Anesthesiologists' overconfidence in their perceived knowledge of neuromuscular monitoring and its relevance to all aspects of medical practice: An international survey. Anesthesia \& Analgesia, 128(6), 1118-1126.

Quinn, B. L., \& Smolinski, M. (2018). Improving school nurse pain assessment practices for students with intellectual disability. The Journal of School Nursing, 34(6), 480-488.

Robertson, J., Hatton, C., Wells, E., Collins, M., Langer, S., Welch, V., \& Emerson, E. (2011). The impacts of short break provision on families with a disabled child: An international literature review. Health \& Social Care in the Community, 19(4), 337-371.

Rosenbaum, P., \& Gorter, J. W. (2012). The 'F-words' in childhood disability: I swear this is how we should think!. Child: Care, Health and Development, 38(4), 457-463.

Sawilowsky, S. S. (2009). New effect size rules of thumb. Journal of Modern Applied Statistical Methods, 8(2), 26.

Tudor, M. E., Walsh, C. E., Mulder, E. C., \& Lerner, M. D. (2015). Pain as a predictor of sleep problems in youth with autism spectrum disorders. Autism, 19(3), 292-300. 
Zhang, C. H., Hsu, L., Zou, B. R., Li, J. F., Wang, H. Y., \& Huang, J. (2008). Effects of a pain education program on nurses' pain knowledge, attitudes and pain assessment practices in China. Journal of Pain and Symptom Management, 36(6), 616-627. 